\title{
A volume-preserving sharpening approach for the propagation of sharp phase boundaries in multiphase lattice Boltzmann simulations
}

\author{
T. Reis*, P. J. Dellar* \\ Oxford Centre for Collaborative Applied Mathematics \\ Mathematical Institute, 24-29 St Giles', OX1 3LB, UK
}

\begin{abstract}
Lattice Boltzmann models that recover a macroscopic description of multiphase flow of immiscible liquids typically represent the boundaries between phases using a scalar function, the phase field, that varies smoothly over several grid points. Attempts to tune the model parameters to minimise the thicknesses of these interfaces typically lead to the interfaces becoming fixed to the underlying grid instead of advecting with the fluid velocity. This phenomenon, known as lattice pinning, is strikingly similar to that associated with the numerical simulation of conservation laws coupled to stiff algebraic source terms. We present a lattice Boltzmann formulation of the model problem proposed by LeVeque and Yee [J. Comput. Phys. 86, 187] to study the latter phenomenon in the context of computational combustion, and offer a volume-conserving extension in multiple space dimensions. Inspired by the random projection method of Bao and Jin [J. Comput. Phys. 163, 216] we further generalise this formulation by introducing a uniformly distributed quasi-random variable into the term responsible for the sharpening of phase boundaries. This method is mass conserving and the statistical average of this method is shown to significantly delay the onset of pinning.
\end{abstract}

Keywords:

lattice Boltzmann method, stiff equations, multiphase flow, pinning, facetting

\section{Introduction}

The lattice Boltzmann method (LBM) is often promoted as a numerical simulation tool that is particularly suitable for predicting complex flows, most notably immiscible fluids in porous media. The lattice Boltzmann approach offers some attractive features to the CFD community, such as a linear constant-coefficient advection operator, and all nonlinearity being confined to algebraic sources terms that may be implemented locally grid point by grid point. This makes the LBM highly amenable to efficient parallel implementations on modern computer architectures. The lattice Boltzmann equation has been successfully applied to a wide variety of flow phenomena $[17,18,19]$ In this paper we focus

${ }^{*}$ Corresponding author

Email addresses: reis amaths.ox.ac.uk (T. Reis), dellar@maths.ox.ac.uk (P. J. Dellar) 
on lattice Boltzmann approaches that simulate two immiscible fluids using a phase field or colour field $\phi$ to represent the different phases, following the work of Gunstensen et al [7] and Granau et al [8]. We do not consider the other approaches for simulating multiphase flow at the molecular level $[9,10,11]$.

Regions where the phase field $\phi \approx 0$ might represent one phase (e.g. oil) and regions where $\phi \approx 1$ the other phase (e.g. water). In principle, $\phi$ is advected by the fluid velocity, hence $\phi_{t}+\mathbf{u} \cdot \nabla \phi=0$. However, to make the most of limited spacial resolution it is common to add a "sharpening term" to counteract the inevitable numerical diffusion and preserve relatively thin boundaries between different phases. For example, the sharpening term might drive all points where $\phi>\frac{1}{2}$ back towards $\phi=1$, and all points where $\phi<\frac{1}{2}$ back towards $\phi=0$. The width of the phase boundary is then controlled by a balance between diffusion and the sharpening term. However, overly narrow phase boundaries can fail to propagate correctly, becoming fixed or "pinned" to the grid $[2,4,5]$.

This combination of advection and sharpening shares all the qualitative properties of the model equation

$$
\phi_{t}+\mathbf{u} \cdot \nabla \phi=S(\phi)=-\frac{1}{T} \phi(1-\phi)\left(\phi-\frac{1}{2}\right)
$$

introduced by LeVeque \& Yee [3] to study the misbehaviour of numerical solutions of hyperbolic equations when the natural timescale $T$ of the source term was small compared with a timestep. Starting from initial conditions representing a step transition from $\phi=1$ to $\phi=0$, equivalent to a boundary between one phase and the other, LeVeque and Yee [3] found that the boundary in their computations either remained stationary, or propagated with the incorrect speed of one grid point per timestep.

Bao \& Jin [1] proposed a random projection method to tackle the difficulties in computing the solutions of stiff hyperbolic conservation laws. Recognising that the effect of solving the partial differential equation (PDE) (1) over a timestep $\Delta t \gg T$ is to project $\phi$ to either 0 or 1 , whichever is closer to its initial value, Bao \& Jin [1] modelled the effect of the source term by an explicit projection to either 0 or 1 at each timestep. However, rather than the direction of the projection being determined always by whether $\phi \gtrless \frac{1}{2}$, they introduced a threshold $\phi_{\mathrm{c}}^{(n)}$ that varied from timestep to timestep. They showed that taking this threshold from the van der Corput sampling sequence gave the correct average propagation speed over many timesteps for a step transition from $\phi=1$ to $\phi=0$.

In an attempt to understand and overcome the phenomenon of pinning in multiphase LBMs, we present a lattice Boltzmann formulation of a random-projection inspired generalisation of the model equation (1). In Section 2 we discuss the problem of stiffness in more detail and summarise the findings of LeVeque \& Yee [3] and Bao \& Jin [1]; in Section 3 we show how one can formulate a lattice 
Boltzmann implementation of the these model problems; and our numerical results are presented in Section 4.

\section{Stiffness and Pinning}

To try and understand the misbehavior of numerical solutions to stiff PDEs, LeVeque and Yee [3] studied the model problem given by equation (1). Algorithms for the advection step (that is, the leftand side of (1)) inevitably introduce a degree of numerical diffusion which leads to some smearing of $\phi$ while the sharpening term $S(\phi)$ drives these intermediate values back to one of its stable equilibria (that is, either 0 or 1), depending on weather the post-advection value is greater or less than the unstable point $\phi_{c}=1 / 2$. Thus, when the system is very stiff, that is when the timestep $\Delta t \gg T$, the sharpening step effectively becomes a deterministic projection of $\phi$ onto its stable equilibrium values:

$$
S_{D}(\phi): \quad \phi(x, t+\Delta t)=\left\{\begin{array}{lll}
1 & \text { if } & \phi^{\prime}(x, t)>1 / 2 \\
0 & \text { if } & \phi^{\prime}(x, t) \leq 1 / 2
\end{array}\right.
$$

where $S_{D}(\phi)$ denotes the discrete operator for the source term and $\phi^{\prime}$ is the post advection value of $\phi$. If $\phi^{\prime}>1 / 2$, the operator defined by equation (2) always drives $\phi$ to one over a timestep, thus forcing the discontinuity to advance by one grid point. Conversely, $\phi \rightarrow 0$ if $\phi^{\prime}<1 / 2$ and the discontinuity does not move at all.

The aim of the random projection method proposed by Bao \& Jin [1] is to explicitly project $\phi$ back to one of its equilibrium values with the direction of projection depending not on the (constant) unstable equilibrium value but rather on the $n^{t h}$ value of uniformly distributed quasi-random sequence $\phi_{c}^{(n)} \in[0,1]$ at the $n^{t h}$ timestep. That is, Bao \& Jin [1] apply the discrete random projection operator $S_{R}$ to the post-advection value of $\phi$, where

$$
S_{R}(\phi): \quad \phi(x, t+\Delta t)=\left\{\begin{array}{lll}
1 & \text { if } & \phi^{\prime}(x, t)>\phi_{c}^{(n)}, \\
0 & \text { if } & \phi^{\prime}(x, t) \leq \phi_{c}^{(n)} .
\end{array}\right.
$$

Bao \& Jin [1] proved that their method yields the correct interface propagation speed with an error of $O(\Delta x|\ln \Delta x|)$ when either the first order upwind or Lax-Friedrichs method is applied to the advection step, and the $\phi_{c}^{(n)}$ are taken from the van der Corput sampling sequence. In other words, the propagation errors that are inevitably incurred at each timestep average out over many timesteps.

The van der Corput sequence is an easily evaluated and equidistributed sequence on the unit interval. If $n \in \mathbb{N}$ has the binary expansion 


$$
n=\sum_{k=0}^{m} \gamma_{k} 2^{k}, \quad \gamma_{k} \in\{0,1\},
$$

the corresponding $n^{\text {th }}$ member of the van der Corput sequence is

$$
\phi_{\mathrm{c}}^{(n)}=\sum_{k=0}^{m} \gamma_{k} 2^{-(k+1)} .
$$

These $\phi_{\mathrm{c}}^{(n)}$ have the properties that $\phi_{\mathrm{c}}^{(n)} \in[0,1], \phi_{\mathrm{c}}^{(1)}=1, \phi_{\mathrm{c}}^{(n)}<1 / 2$ for $n$ even, and $\phi_{\mathrm{c}}^{(n)}>1 / 2$ for $n$ odd.

Inspired by these findings, we generalise the model partial differential equation (1) studied by LeVeque \& Yee [3] to

$$
\phi_{t}+\mathbf{u} \cdot \nabla \phi=S(\phi)=-\frac{1}{T} \phi(1-\phi)\left(\phi-\phi_{\mathrm{c}}^{(n)}\right)
$$

In other words, we replace the previous constant threshold $\phi_{c}=1 / 2$ in the sharpening term by a member of the van der Corput sequence. We use the $n^{\text {th }}$ member of the sequence at the $n^{\text {th }}$ timestep for all grid points.

\section{Lattice Boltzmann Formulation}

We now construct a lattice Boltzmann formulation for our model equation (6), following the usual approaches for advection-reaction-diffusion equations $[12,13,14,15]$. We express the phase field $\phi$ as the zeroth moment of a set of distribution functions $f_{i}(x, t)$,

$$
\phi=\sum_{i} f_{i}
$$

where the $\mathbf{c}_{i}$ are a given set of discrete velocities. We then postulate the discrete Boltzmann equation

$$
\partial_{t} f_{i}+\mathbf{c}_{i} \cdot \nabla f_{i}=-\frac{1}{\tau}\left(f_{i}-f_{i}^{(0)}\right)+R_{i}
$$

The equilibrium distributions are

$$
f_{i}^{(0)}=w_{i} \phi\left(1+\theta^{-1} \mathbf{c}_{i} \cdot \mathbf{u}\right)
$$

where $\mathbf{u}$ is the advection velocity, which we assume to be constant. The $w_{i}$ are constant weights associated with the particle velocities $\mathbf{c}_{i}$. Examples of these are given below. The lattice constant $\theta$ is defined by

$$
\sum w_{i} c_{i \alpha} c_{i \beta}=\theta \delta_{\alpha \beta}
$$


where Greek indices refer to Cartesian components, and repeated Greek indices will be summed. Sharpening is provided by the term $R_{i}$, which must satisfy

$$
\sum_{i} R_{i}=S(\phi)
$$

\subsection{Macroscopic limit}

As is standard in the lattice Boltzmann approach, we aim to recover our model equation (6) as the equation describing slowly varying solutions of (8), i.e. solutions that vary over timescales much longer than the collision time $\tau$. Taking he first two moments of (8) gives

$$
\begin{aligned}
\partial_{t} \phi+\nabla \cdot \boldsymbol{\psi} & =S(\phi) \\
\partial_{t} \boldsymbol{\psi}+\nabla \cdot \boldsymbol{\Pi} & =-\frac{1}{\tau}\left(\boldsymbol{\psi}-\boldsymbol{\psi}^{(0)}\right)+\mathbf{G}(\phi),
\end{aligned}
$$

where higher moments of the $f_{i}$ and $R_{i}$ are defined by

$$
\begin{aligned}
\psi & =\sum_{i} f_{i} \mathbf{c}_{i}, \\
\mathbf{G}(\phi) & =\sum_{i} R_{i} \mathbf{c}_{i}, \\
\Pi & =\sum_{i} f_{i} \mathbf{c}_{i} \mathbf{c}_{i} .
\end{aligned}
$$

We seek the slowly varying behaviour using the multiple-scales version of a Chapman-Enskog expansion.

We expand the non-conserved moments $\psi, \Pi$, and the time derivative $\partial_{t}$ as series in the collision time $\tau$ :

$$
\boldsymbol{\psi}=\boldsymbol{\psi}^{(0)}+\tau \boldsymbol{\psi}^{(1)}+\cdots, \boldsymbol{\Pi}=\Pi^{(0)}+\tau \Pi^{(1)}+\cdots, \partial_{t}=\partial_{t_{0}}+\tau \partial_{t_{1}} \cdots
$$

We do not expand $\phi$ because it is conserved by the collision term. The leading order contributions to the fluxes are

$$
\boldsymbol{\psi}^{(0)}=\mathbf{u} \phi, \quad \boldsymbol{\Pi}=\theta \phi \mathbf{I}
$$

where $\mathbf{I}$ is the identity tensor. Substituting these into equation (13), and evaluating $\partial_{t_{0}} \boldsymbol{\psi}^{(0)}$ using the leading order approximation to equation (12), yields an expression for the first correction $\boldsymbol{\psi}^{(1)}$ to the flux, 
We choose $\mathbf{G}(\phi)=\mathbf{u} S(\phi)$ so that, after a little algebra,

$$
\boldsymbol{\psi}=\mathbf{u} \phi-\tau(\theta \nabla \phi-\mathbf{u}(\mathbf{u} \cdot \nabla) \phi)+O\left(\tau^{2}\right)
$$

Substituting this expression for the flux into the evolution equation (12) for $\phi$ yields the advection/diffusion/reaction equation

$$
\frac{\partial \phi}{\partial t}+\mathbf{u} \cdot \nabla \phi=S(\phi)+\tau\left(\theta \nabla^{2}-\mathbf{u u}: \nabla \nabla\right) \phi+O\left(\tau^{2}\right)
$$

The leading order terms match our intended equation (6). The inevitable diffusive correction term at $O(\tau)$ will set the width of the boundary between the two fluids. A sharpening term $R_{i}$ that satisfies the constraints $\sum_{i} R_{i}=S(\phi)$ and $\sum_{i} R_{i} \mathbf{c}_{i}=\mathbf{G}(\phi)=\mathbf{u} S(\phi)$ is

$$
R_{i}=-w_{i} S(\phi)\left[1+\theta^{-1} \mathbf{c}_{i} \cdot \mathbf{u}\right]
$$

analogous to the expression (9) for the $f_{i}^{(0)}$.

\subsection{Reduction to fully discrete form}

To solve (8) numerically we must discretise it in space and time. This may be achieved by integrating (8) along a characteristic for a time $\Delta t$ to obtain

$$
f_{i}\left(x+c_{i} \Delta t, t+\Delta t\right)-f_{i}(x, t)=\int_{0}^{\Delta t} \Omega_{i}\left(x+c_{i} s, t+s\right) d s,
$$

where $\Omega_{i}$ represents the combination of collision and source terms,

$$
\Omega_{i}(x, t)=-\frac{1}{\tau}\left(f_{i}(x, t)-f_{i}^{(0)}(x, t)\right)+R_{i}(x, t) .
$$

The left hand side of (8) is a derivative along a characteristic, so the left hand side of (23) is exact. The remaining integral on the right hand side may be approximated by the trapezium rule to yield the second-order accurate but implicit system of algebraic equations

$$
\begin{aligned}
f_{i}\left(x+c_{i} \Delta t, t+\Delta t\right)-f_{i}(x, t) & =\frac{\Delta t}{2}\left(\Omega_{i}\left(x+c_{i} \Delta t, t+\Delta t\right)\right. \\
& \left.+\Omega_{i}(x, t)\right)+O\left(\Delta t^{3}\right)
\end{aligned}
$$

Following He et al. [6], we now introduce the new variables

$$
\bar{f}_{i}\left(x^{\prime}, t^{\prime}\right)=f_{i}\left(x^{\prime}, t^{\prime}\right)-\frac{\Delta t}{2 \tau}\left(f_{i}\left(x^{\prime}, t^{\prime}\right)-f_{i}^{(0)}\left(x^{\prime}, t^{\prime}\right)\right)-\frac{\Delta t}{2} R_{i}\left(x^{\prime}, t^{\prime}\right)
$$


Using these variables, the previous implicit scheme (25) rearranges into explicit formulae for the $\bar{f}_{i}$ at the new timestep,

$$
\begin{aligned}
\bar{f}_{i}\left(x+c_{i} \Delta t\right. & , t+\Delta t)-\bar{f}_{i}(x, t) \\
= & -\frac{\Delta t}{\tau+\Delta t / 2}\left(\bar{f}_{i}(x, t)-f_{i}^{(0)}(x, t)\right)+\frac{\tau \Delta t}{\tau+\Delta t / 2} R_{i}(x, t) .
\end{aligned}
$$

We thus discard the $f_{i}$ and evolve the $\bar{f}_{i}$ instead using (27). However, we still require the phase field $\phi$, which is defined in terms of the $f_{i}$ rather than the $\bar{f}_{i}$. By taking the zeroth moment of (26) we find

$$
\begin{aligned}
\bar{\phi}=\sum_{i} \bar{f}_{i} & =\sum_{i}\left[f_{i}-\frac{\Delta t}{2 \tau}\left(f_{i}-f_{i}^{(0)}\right)-\frac{\Delta t}{2} R_{i}\right] \\
& =\phi+\frac{\Delta t}{2 T} \phi(1-\phi)\left(\phi_{c}^{(n)}-\phi\right)=N(\phi) .
\end{aligned}
$$

We must solve the cubic equation $N(\phi)=\bar{\phi}$ to recover $\phi$, which is need to evaluate $f_{i}^{(0)}$ and $R_{i}$. This may be achieved using a few iterations of Newton's method,

$$
\phi \rightarrow \phi-\frac{N(\phi)-\bar{\phi}}{N^{\prime}(\phi)} .
$$

In all the numerical experiments we've conducted, satisfactory convergence took at most four iterations starting from $\bar{\phi}=\phi$. Notice that $N(\phi)=\phi+O(\Delta t / T)$, so the function $N$ is close to linear when $\Delta t \ll T$. This difficulty is not normally present in lattice Boltzmann formulations for hydrodynamics, because the quantities $\rho$ and $\mathbf{u}$ needed to evaluate the $f_{i}^{(0)}$ are conserved under collisions.

Another subtle complication arises because the collision term $R_{i}$ used in the definition of $\bar{f}_{i}$ varies from timestep to timestep through its dependence on $\phi_{c}^{(n)}$. At each timestep we must convert from $\bar{f}_{i}$ to $f_{i}$ using the threshold from the previous timestep, then from these $f_{i}$ back to $\bar{f}_{i}$ using the new threshold for the current timestep.

\section{Numerical Experiments}

The width and propagation speed of phase boundaries governed by the advection/diffusion/reaction equation (21) is controlled by the timescale $T$ of the sharpening term, and the timescale $\tau$ of the collision term controlling numerical diffusion. The phase boundary extends across $O\left((\tau T)^{1 / 2}\right)$ grid points, as measured in the so-called lattice units where $\partial_{x}=\partial_{t}=1$. The propagation speed is controlled by the timescale ratio $\tau / T$. 
In Figure 1 we show the measured propagation speeds of interfaces in one-dimensional computations using both the constant and van der Corput thresholds. Results for the constant threshold are shown for both the D1Q2 and D1Q3 lattices. The former uses two particles with speeds $c_{ \pm}= \pm 1$, weights $w_{ \pm}=1 / 2$ and lattice constant $\theta=1$. The latter adds an additional rest particle $c_{0}=0$, and changes the weights to $w_{+}=w_{-}=1 / 6$ and $w_{0}=1 / 3$. The lattice constant is $\theta=1 / 3$. The deterministic D1Q3 model offers some improvement over the D1Q2 model, especially for smaller values of $\tau / T$, but both models suffer a reduction in propagation speed due to $\tau / T$. When $\tau / T$ becomes sufficiently large the interface no longer propagates and becomes pinned to the grid. By contrast, the D1Q3 model using the van der Corput thresholds $\phi_{\mathrm{c}}^{(n)}$, instead of the constant threshold $\phi=1 / 2$ for all $n$, preserves the approximately correct propagation speed even for large values of $\tau / T$.

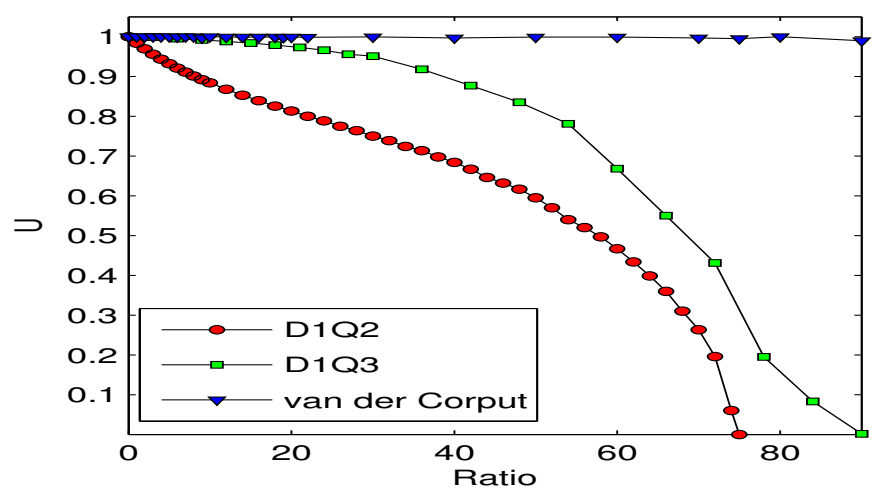

Figure 1: Interface propagation speeds as a function of the timescale ratio $\tau / T$. The random-threshold method maintains the correct propagation speed even when $\tau / T \gg 1$.

The model extends easily to two dimensions using the standard D2Q9 lattice with nine velocities, and the following expressions for the equilibrium functions and source terms,

$$
\begin{aligned}
f_{i}^{(0)} & =w_{i} \phi\left(1+3 \mathbf{c}_{i} \cdot \mathbf{u}\right), \\
R_{i} & =w_{i} S(\phi)\left(1+3 \mathbf{c}_{i} \cdot \mathbf{u}\right) .
\end{aligned}
$$

The $w_{i}$ are the usual D2Q9 lattice weights, $w_{0}=4 / 9, w_{1,2,3,4}=1 / 9$, and $w_{5,6,7,8}=1 / 36$ [16].

Figure 2 shows the evolution of an initially circular patch under the two-dimensional scheme with constant threshold $\phi_{c}=1 / 2$, advection velocity $\mathbf{u}=(1,1)$, and timescale ratio $\tau / T=10$. The patch shrinks and eventually disappears. This loss of mass is caused by the curvature of the interface, which is a new phenomenon that was not present in the earlier one-dimensional simulations. Consider a circular patch of fluid as represented by the sketch in Figure 3. Well inside the patch $\phi=1$, and well outside the patch $\phi=0$. The grey areas indicate interfacial regions, the darker have a value 

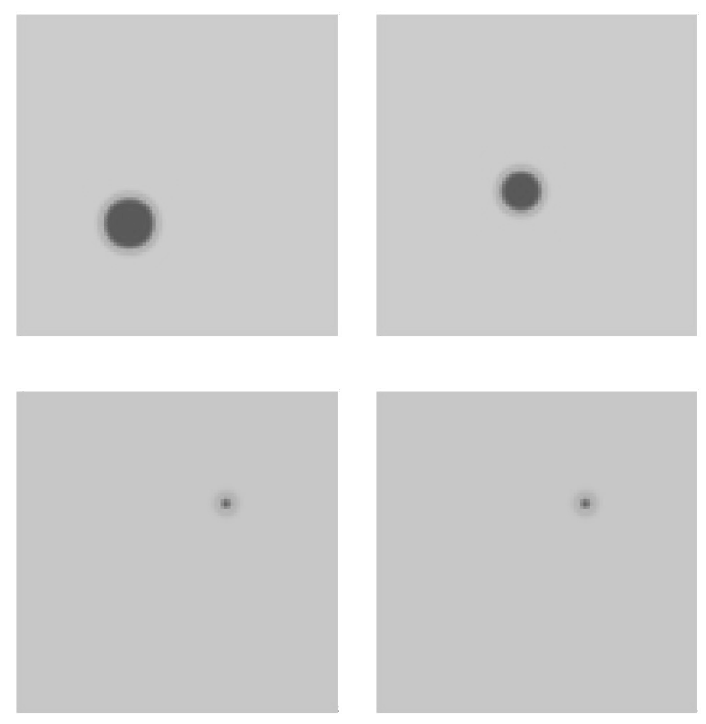

Figure 2: Example of area loss using the scheme defined in (27) with $\tau / T=10$.

of the phase field in the range $1 / 2<\phi<1$, and the lighter having $\phi$ in the range $0 \leq \phi<1 / 2$. The outer region covers a larger area on the plane than the inner region, so if we apply a constant threshold $\phi_{\mathrm{c}}=1 / 2$, or even a sequence of thresholds $\phi_{\mathrm{c}}^{(n)}$ with average value $\left\langle\phi_{\mathrm{c}}^{(n)}\right\rangle=1 / 2$, slightly more $\phi$ is driven towards zero by the sharpening term in the outer annulus than is driven towards 1 by the sharpening term in the inner annulur. This imbalance, which is proportional to the curvature, is responsible for the shrinkage of the patch shown in Figure 2. A similar mass leakage affects level set methods, and for the same reason.

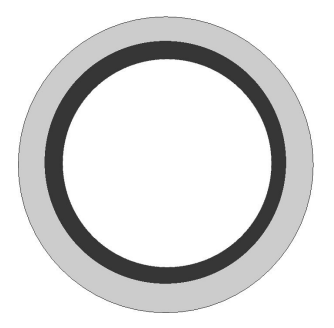

Figure 3: Sketch representing the mechanism behind the loss of area. The outer annulus covers a slightly larger area than the inner when the average threshold $\left\langle\phi_{c}^{(n)}\right\rangle=1 / 2$.

One way to cure the mass leakage problem is to replace the previous threshold $\phi_{c}^{(n)}$ with a threshold $\phi_{M}^{(n)}$ chosen to conserve volume. Since the diffused $\phi$ is smooth, the volume $V(\chi)=\operatorname{Volume}(\{\mathbf{x}$ : $\phi<\chi\})$ enclosed by a level set $\chi$ is a monotonically increasing, and hence invertible function, of $\chi$. There is thus a unique volume-preserving $\phi_{M}^{(n)}$ at each timestep, such that $V\left(\phi_{M}^{(n)}\right)=V_{0}$ equals the volume of the patch at the start of the timestep. This amounts to finding at each timestep the $\phi_{M}^{(n)}$ that 
satisfies the following integral constraint over the domain $\Omega$.

$$
\int_{\Omega} S\left(\phi, \phi_{M}^{(n)}\right)=-\int_{\Omega} \frac{1}{T} \phi(1-\phi)\left(\phi-\phi_{M}^{(n)}\right)=0
$$

where $S\left(\phi, \phi_{M}^{(n)}\right)$ denotes the source term with a parametric dependence on $\phi_{M}^{(n)}$. To incorporate a degree of quasi-randomness into this method the van der Corput sequence and its range may be scaled so that its mean value is the volume-conserving level set.

Figure 4 shows the evolution of an initially circular patch under the lattice Boltzmann scheme using the volume-preserving threshold obtained from (32) at each timestep. The applied velocity was $\mathbf{u}=(1,1)$, and the timescale ratio was $\tau / T=50$. The patch undergoes severe facetting, and becomes pinned to the computational grid. The results of the analogous computations using the same parameters and the biased mass-conserving van der Corput sequence are plotted in Figure 5. They show that the patch propagates as expected, while maintaining a circular shape. These results are confirmed by the scatter plot of $\phi$ versus distance from the centre of the patch in Figure 6 . The random projection approach maintains a close to circular patch with a thin interface spanning no more than 3 lattice points.
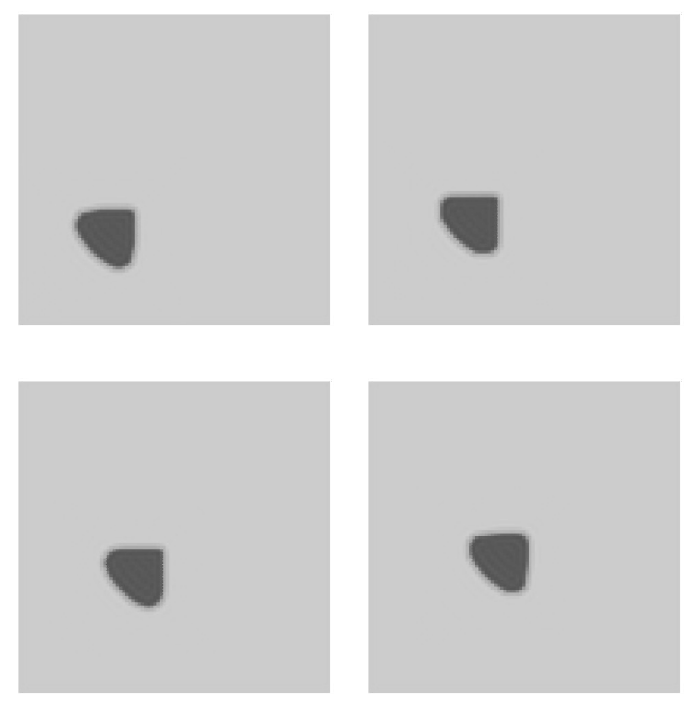

Figure 4: Propagating circular patch with timescale ratio $\tau / T=50$. Plots from left to right correspond to $t=$ $0.1,0.2,0.3,0.4$.

\section{Conclusion}

We have presented a volume-preserving multi-dimensional lattice Boltzmann implementation of a generalisation of the model equation studied by LeVeque and Yee [3]. Our model incorporates a quasi-random threshold to overcome the problems of numerical stiffness, as proposed by Bao and 

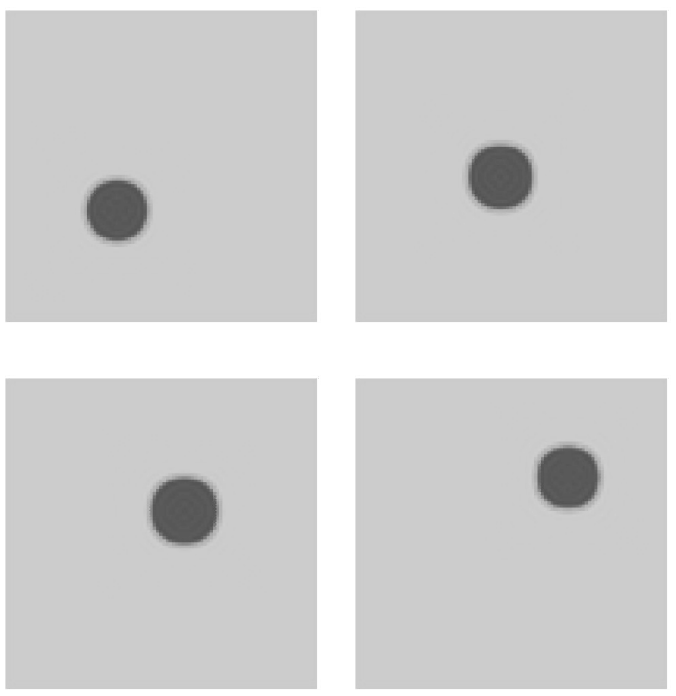

Figure 5: Propagating circular patch with timescale ratio $\tau / T=50$. Plots from left to right correspond to $t=$ $0.1,0.2,0.3,0.4$.

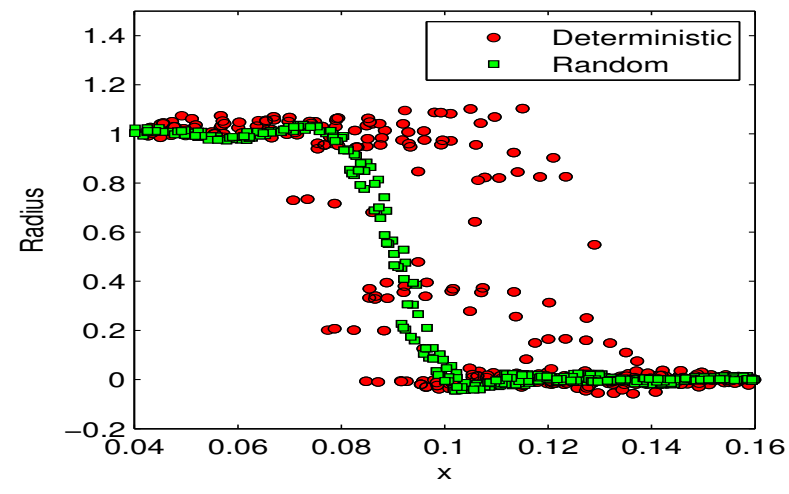

Figure 6: Scatter plot of $\phi$ when $\tau / T=50$ using a deterministic (०) and random ( $\square$ ) type models. The random threshold approach offers a much closer approximation to an isotropic solution. 
Jin [1]. We further modified the quasi-random threshold by applying a bias to the distribution of thresholds that corrects for the mass leakage associated with curvature of the interface. This method has been shown to predict the correct propagation speed of sharp fronts, while conserving volume in simple two-dimensional experiments. It would be viable for simulations of suspensions of separated droplets, in conjunction with a tracking algorithm to identify volumes containing individual droplets. However, due to the difficulty of computing suitable mass-conserving thresholds, it is unlikely to be appropriate for multi-phase computations in which droplets undergo mergers or fission.

\section{Acknowledgments}

The authors' research is supported by Award No. KUK-C1-013-04, made by King Abdullah University of Science and Technology (KAUST); and by an Advanced Research Fellowship, grant number EP/E054625/1, from the UK's Engineering and Physical Sciences Research Council (EP$\mathrm{SRC})$.

[1] W. Bao and S. Jin, The random projection method for hyperbolic conservation laws with stiff reaction terms,

J. Comput. Phys., 163 (2000), 216-248.

[2] M. Latva-Kokko and D. H. Rothman, Diffusion properties of gradient-based lattice Boltzmann models of immiscible fluids, Phys. Rev. E, 71 (2005), 056702

[3] R. J. LeVeque and H. C. Yee, A study of numerical methods for hyperbolic conservation laws with stiff source terms, J. Comput. Phys., 86 (1990), 187-210

[4] I. Halliday, R. Law, C. M. Care and A. Hollis, Improved simulation of drop dynamics in a shear flow at low Reynolds and capillary number, Phys. Rev. E, 73 (2006), 056708

[5] J. Tölke, S. Freudiger and M. Krafczyk, An adaptive scheme using hierarchial grids for lattice Boltzmann multi-phase flow simulations, Comput. Fluids, 35 (2006), 820-830

[6] X. He, X. Shan and G. D. Doolen, Discrete Boltzmann equation model for nonideal gases, Phys. Rev. E, 57 (1998), R13-R16

[7] A. Gunstensen and D. Rothman and S. Zaleski and G. Zanetti, Lattice Boltzmann model of immiscible fluids, Phys. Rev. A, 43 (1991), 4320-4327

[8] D. Grunau and S. Chen and K. Eggert, A lattice Boltzmann model for multiphase fluid flows, Phys. Fluids. A, 5 (1993), 2557-2562 
[9] H. Chen and X. Shan, Lattice Boltzmann model for simulating flows with multiphase components, Phys. Rev. E, 47 (1993), 1815-1819

[10] M. Swift and W. Osbourne and J. Yeomans, Lattice Boltzmann simulations for non-ideal fluids, Phys. Rev. Lett, 75 (1995), 830-833

[11] E. Aurell and D.-Q. Minh, An inventory of Lattice Boltzmann models of multiphase flows, eprint cond-mat/0105372, 2001

[12] D. Wolf-Gladrow, A lattice Boltzmann equation for diffusion, J. Stat. Phys, 79 (1995), 1023 1032

[13] Z. L. Guo and B. C. Shi and N. C. Wang, Fully Lagrangian and Lattice Boltzmann Methods for the Advection-Diffusion Equation, J. Sci. Comput., 14 (1999), 291-300

[14] S. P. Dawson and S. Chen and G. D. Doolen, Lattice Boltzmann computations for reactiondiffusion equations, J. Chem. Phys. 98 (1993), 1514

[15] A. Hiorth and U. H. a Lad and S. Evje and S. M. Skjaeveland, A Lattice Boltzmann-BGK algorithm for a diffusion equation with Robin boundary condition - application to NMR relaxation, Int. J. Numer. Meth. Fluids, 59 (2009), 405-421

[16] Y. H. Qian and D. d'Humiéres and P. Lallemand, Lattice BGK Models for Navier-Stokes Equation, Europhys. Lett., 17 (1992) 479

[17] S. Succi, The Lattice Boltzmann Equation for Fluid Dynamics and Beyond, Oxford Univeristy Press, 2001

[18] S. Chen and G.D. Doolen, Lattice Boltzmann method for fluid flows, Ann. Rev. Fluid. Mech., 30 (1998), 329-364

[19] D. Yu and R. Mei and L. Luo and W. Shyy, Viscous flow computations with the method of lattice Boltzmann equation, Prog. Aero. Sci 39 (2003) 329-367 